\title{
Controlling Nanoparticles Formation in Molten Metallic Bilayers by Pulsed-Laser Interference Heating
}

\author{
M. Khenner ${ }^{1 *}$, S. Yadavali ${ }^{2}$, R. Kalyanaraman ${ }^{3}$ \\ ${ }^{1}$ Department of Mathematics, Applied Physics Institute, \\ Western Kentucky University, Bowling Green, KY 42101 \\ ${ }^{2}$ Department of Chemical and Biomolecular Engineering, \\ The University of Tennessee, Knoxville, TN 37996 \\ ${ }^{3}$ Department of Chemical and Biomolecular Engineering, Department of Materials Science \\ and Engineering, Sustainable Energy Education Research Center, \\ The University of Tennessee, Knoxville, TN 37996
}

\begin{abstract}
The impacts of the two-beam interference heating on the number of core-shell and embedded nanoparticles and on nanostructure coarsening are studied numerically based on the non-linear dynamical model for dewetting of the pulsed-laser irradiated, thin $(<20 \mathrm{~nm})$ metallic bilayers. The model incorporates thermocapillary forces and disjoining pressures, and assumes dewetting from the optically transparent substrate atop of the reflective support layer, which results in the complicated dependence of light reflectivity and absorption on the thicknesses of the layers. Stabilizing thermocapillary effect is due to the local thickness-dependent, steadystate temperature profile in the liquid, which is derived based on the mean substrate temperature estimated from the elaborate thermal model of transient heating and melting/freezing. Linear stability analysis of the model equations set for $\mathrm{Ag} / \mathrm{Co}$ bilayer predicts the dewetting length scales in the qualitative agreement with experiment.
\end{abstract}

Keywords and phrases: liquid bilayer films, thermocapillary convection, interfacial stability, pulsed laser irradiation, dewetting, self-organization, nanopatterning

Mathematics Subject Classification: 76D08, 76D45, 76E17, 76E30, 37N10

\section{Introduction}

Accurate mathematical models that capture natural or spontaneous processes that have technological implications are important for a deeper understanding and optimization of the process. One such technique that is being harnessed to create complex nanostructures of metals and polymers is the spontaneous spinodal dewetting of thin liquid films from its contact surface [1-3]. For an ultrathin film on a nonwetting substrate, long-range intermolecular attractive dispersion forces between the film and substrate, with magnitude determined by the Hamaker coefficient $A$, lead to an unstable thermodynamic free energy

${ }^{*}$ Corresponding author. E-mail: mikhail.khenner@wku.edu. 
which can have a spinodal character, i.e. the free energy curvature $<0$. Consequently, for such thin films, a height perturbation of the liquid film surface, such as by capillary waves, can result in a free energy decrease, leading to spontaneous spinodal dewetting $[1,2]$. In order to observe this behavior in metals, which have extremely high melting temperatures, practical ways to access the liquid phase are necessary. Therefore, studies of pattern formation in metal films have focused on the use of ion irradiation $[4,5]$ or pulsed laser irradiation [6-8].

Laser-irradiation of metallic films exhibit a variety of spatio-temporal instabilities leading to formation of different nanomorphologies [9-15]. This applied modeling paper is motivated by the recent experiments on dewetting of non-reactive, immiscible metallic bilayers [16], and the need to better understand the physical mechanisms leading to spatial ordering of particles and the morphological pathways towards the final particle morphologies. The experimental [17-24] and theoretical [25-34] studies of the instabilities and dynamics of bilayer thin films so far have been largely limited to aqueous and polymer systems under isothermal conditions. Non-isothermal dewetting even in such more conventional systems has not been fully investigated theoretically. Indeed, in Refs. $[25,26]$ the equation set that accounts for the Marangoni effect is derived, but only the analysis and some computations in the isothermal case are performed; and in Refs. [32-34] either a special set of Hamaker coefficients is considered, such that dewetting exhibits autophobic behavior, or in addition a longitudinal temperature gradient is considered. Also, to our knowledge, even in the isothermal case no combined experimental/theoretical study has been published and thus the theory has not been quantitatively matched to an experiment.

We are motivated to investigate liquid bilayers due to the need to create nanomaterials that integrate multiple functionalities. The applications of such materials can be widespread. For instance, plasmonic - ferromagnetic materials can be used to improve chemical sensing time scales [35-37], quantify disease detection [38-40], and improve high-speed optical communication circuits [41,42]. However, existing synthesis routes utilizing thin film or liquid routes have found it difficult to independently control the nanoparticle size and composition over a wide range [43-45]. We have shown recently that it is possible to achieve systematic control on size (between $25-350 \mathrm{~nm}$ ) as well as the ratio of the metals in the particle by single beam laser dewetting of $\mathrm{Ag} / \mathrm{Co}$ bilayer liquids [16]. However, two-beam interference of bilayer liquids can introduce new structures, such as nanowires and particles with elliptical shapes, thus providing additional ways to tune the physical properties of these materials [15]. Therefore, a detailed study of the evolution of the morphology and structures of the bilayer liquids will be important towards eventual studies that correlate the physical properties of the ensuing nanomaterials and their dependence on shape and structure.

We note also that the influence of radiative heating on film stability and dynamics was theoretically studied only in a handful of papers [46-51]. Oron \& Peles [49] studied thermocapillary flows and instabilities of evaporative thin aqueous films with constant internal heat generation. Oron [50] expanded this study to the case of irradiation by a single continuous-wave laser beam. Grigoriev [51] analyzed mechanisms of passive and active feedback control of evaporatively driven instabilities in irradiated thin films. Also, Ajaev and Willis [46] studied axisymmetric dewetting and rupture, in a molten state, of a thin metallic film, which has been melted by a single energetic laser pulse with a Gaussian spatial shape. They identified the thermocapillary stresses as the major driving force of a film evolution. It must be noted that neither of these papers considers nonuniform irradiation or the film reflectivity. The latter, as has been pointed out in Refs. $[8,12]$ is often the quantity of key importance for the dynamics of the ultrathin metallic films. Other relevant works include modeling of break-up into nanoparticles of pulsed-laser melted narrow lines and rings [52-54]. In such systems, Rayleigh-Plateau, contact line and line edge instabilities (which are not associated with the temperature field) arise from the outset and seem largely responsible for the film evolution and the resultant length and time scales.

Pulsed laser irradiation of single-layer metal thin films has been shown to introduce thermocapillary effects along the film - ambient surface and thus influence the pattern formation in single layer films $[12,55,56]$. The primary reason for the appearence of the thermocapillary effect is a non-trivial (i.e., nonlinear) dependence of the temperature on film thickness, which is due to the thickness-dependent laser 
light reflectivity and absorption [12,55]. In Ref. [56] a set of coupled, dimensionless evolution equations for the film height and film temperature is derived from the Navier-Stokes equations in the lubrication approximation, following the procedure outlined in Ref. [57] and for the parameters range corresponding to specific thermal conditions of a thin, laser-melted metallic films. Stability and dewetting of such single-layer films is analyzed and computed. Building upon this work, the non-isothermal instabilities and morphological evolution in laser-melted bilayers are studied in Ref. [58]. In this companion paper, we focus on the $\mathrm{Ag} / \mathrm{Co}$ bilayer system and perform a computational study in order to elucidate the effects on the inter-particle spacing and morphologies of the laser heating in the interference mode. Also, using the linear stability analysis, we further explore the length scales of the pattern of droplets that results upon dewetting in the absence of interference.

Detailed experiments of two-beam interference irradiation of single-layer Co films on $\mathrm{SiO}_{2}$ substrates have been reported in Refs. $[14,15]$. These experiments demonstrate that the typical pattern evolution for films thicker than $2 \mathrm{~nm}$ begins with spatially periodic film rupture at length scales equal to the interference spacing at the early stages. Longer irradiation yielded the formation of long, cylindrical-like "nanowires" and continued irradiation resulted in the breakup of these nanowires into particles. The final particle state is characterized as a quasi-2D array, featuring the long-range order due to periodic laser intensity and the short-range order resulting from the breakup of the nanowires. Because of the generally low vapor pressures of metals and the short time scales of heating under the laser pulse, we have observed that evaporation is negligible for the typical conditions of the experiment. The amount of evaporation is measured experimentally by detecting the total amount of remaining material using x-ray analysis in a scanning electron microscope. Typically, experimentally measured evaporation losses are less then $15 \%$ over 10,000 pulses, a typical upper limit on the number of laser pulses applied [11]. For this reason, it is reasonable to assume that evaporation does not play a major role in the typical laser-metal experiments discussed here. The 1D linear stability analysis and computations in this paper predict the long-range order only, i.e. analogous to the spacing between nanowires in experiment. Similar computation for a single-layer film has been reported in Ref. [56].

\section{Problem statement}

\subsection{Evolution equations for the bilayer}

We consider a 2D bilayer thin film (Figure 1(a)), which is comprised of two superposed layers of immiscible Newtonian liquids, for instance the molten Co and Ag. The lower layer rests on a solid, planar substrate (denoted by the subscript $s$ hereafter) that has thickness $h_{s}$, and the upper layer is in contact with the adjacent gas phase. Beneath the substrate $\left(\mathrm{SiO}_{2}\right.$ in the experiments) there is a support layer of $\mathrm{Si}$, whose thickness is much larger than $h_{s} . \mathrm{SiO}_{2}$ is transparent to laser irradiation, while $\mathrm{Si}$ is reflective. The locations of the liquid-liquid and the liquid-gas interfaces are given by $z=h_{1}(x, t)$ and $z=h_{2}(x, t)$, respectively, where $z, x$ are the vertical and the horizontal coordinates, respectively, with $z=0$ corresponding to the location of substrate/liquid inteface. The bilayer is irradiated either by a single pulsed laser beam, or by two laser beams in the interference mode. The pulse width typically is $10 \mathrm{~ns}$, and the frequency of pulses is $50 \mathrm{~Hz}$. Evaporation is negligible.

In the case of a single-layer film, dewetting has been successfully modeled as continuous in time by treating the film as liquid despite that it re-solidifies after each pulse. Thermocapillary effect owing to latent heat release is negligible due to extremely large cooling rates involved [8]; the film cools down so fast that there is insufficient time for any morphology change [59], and the next incident laser pulse effectively quenches in the film. The time and length scales involved and the resulting morphologies are captured very well by such models $[8,12,56]$. In this paper the same strategy is adopted for the bilayer film modeling.

Since the lubrication approximation has been justified in Ref. [56], here we start directly from the non-isothermal system of lubrication equations, where the thermocapillary effect is accounted for at the 


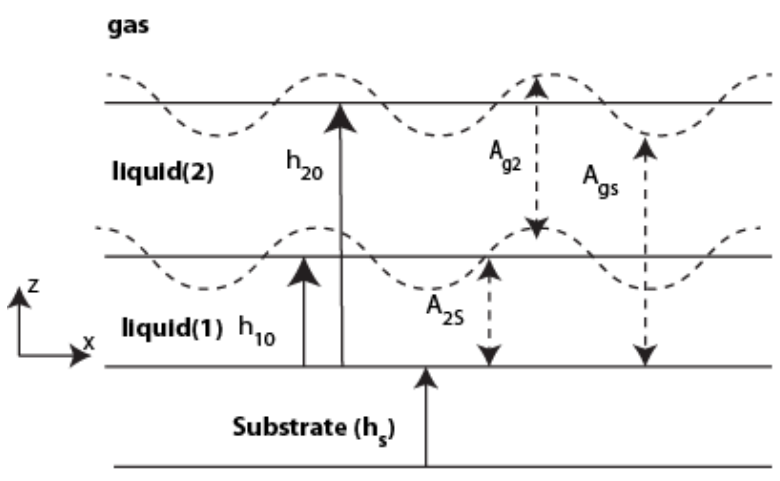

(a)

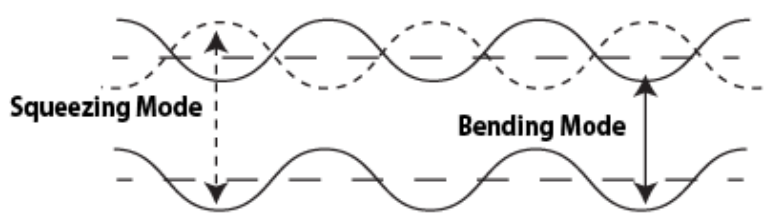

(b)

FiguRE 1. (a) Liquid bilayer/substrate system. Here $A_{g 2}, A_{g s}$ and $A_{2 s}$ are Hamaker coefficients of the intermolecular repulsion or attraction force, $h_{10}, h_{20}$ are the unperturbed thicknesses of the liquid layers, and $h_{s}$ is the thickness of $\mathrm{SiO}_{2}$ substrate. $\mathrm{Si}$ support layer is not shown. (b) Deformation modes of the two interfaces.

liquid-liquid and liquid-gas interfaces [25-27,32,33]:

$$
\begin{aligned}
\partial_{t} h_{1} & =-\partial_{x}\left[F_{11} \partial_{x} P_{1}+F_{12} \partial_{x} P_{2}+\Phi_{11} \partial_{x} \sigma_{1}+\Phi_{12} \partial_{x} \sigma_{2}\right], \\
\partial_{t} h_{2} & =-\partial_{x}\left[F_{21} \partial_{x} P_{1}+F_{22} \partial_{x} P_{2}+\Phi_{21} \partial_{x} \sigma_{1}+\Phi_{22} \partial_{x} \sigma_{2}\right],
\end{aligned}
$$

where $P_{1,2}$ are the pressures and $\sigma_{1,2}$ are the surface tensions of the liquid-liquid and liquid-gas interface, respectively. (Hereafter, the notation $f_{\alpha, \beta}$ means $f_{\alpha}, f_{\beta}$.) The quantities $F_{i j}$ and $\Phi_{i j}, i, j=1, . ., 2$ are given by:

$$
\begin{aligned}
& F_{11}=-\frac{1}{3 \eta_{1}} h_{1}^{3}, F_{12}=-\frac{1}{2 \eta_{1}} h_{1}^{2}\left(h_{2}-\frac{h_{1}}{3}\right), \\
& F_{21}=\frac{1}{6 \eta_{1}} h_{1}^{3}-\frac{1}{2 \eta_{1}} h_{1}^{2} h_{2}, F_{22}=\frac{1}{3}\left(h_{2}-h_{1}\right)^{3}\left(\frac{1}{\eta_{1}}-\frac{1}{\eta_{2}}\right)-\frac{1}{3 \eta_{1}} h_{2}^{3}, \\
& \Phi_{11}=\Phi_{12}=\frac{1}{2 \eta_{1}} h_{1}^{2}, \Phi_{21}=-\frac{1}{2 \eta_{1}} h_{1}\left(h_{1}-2 h_{2}\right), \\
& \Phi_{22}=\frac{1}{2 \eta_{2}}\left(h_{2}-h_{1}\right)^{2}+\Phi_{21},
\end{aligned}
$$

where $\eta_{1,2}$ are the dynamic viscosities. The pressures are:

$$
\begin{aligned}
& P_{1}=-\sigma_{1} \partial_{x x} h_{1}-\sigma_{2} \partial_{x x} h_{2}+\Pi_{1}\left(h_{1}, h_{2}\right)+\rho_{1} g h_{1}+\rho_{2} g\left(h_{2}-h_{1}\right), \\
& P_{2}=-\sigma_{2} \partial_{x x} h_{2}+\Pi_{2}\left(h_{1}, h_{2}\right)+\rho_{2} g h_{2},
\end{aligned}
$$

where $\rho_{1,2}$ are the densities, $g$ is the acceleration of gravity, and $\Pi_{1,2}\left(h_{1}, h_{2}\right)$ are the disjoining pressures, given by

$$
\begin{aligned}
& \Pi_{1}\left(h_{1}, h_{2}\right)=\frac{A_{2 s}}{h_{1}^{3}}+\frac{A_{g s}}{h_{2}^{3}}+\frac{S_{1} \exp \left(-\frac{h_{1}}{\ell_{1}}\right)}{l_{1}}, \\
& \Pi_{2}\left(h_{1}, h_{2}\right)=\frac{A_{g 2}}{\left(h_{2}-h_{1}\right)^{3}}+\frac{A_{g s}}{h_{2}^{3}}+\frac{S_{2} \exp \left(-\frac{\left(h_{2}-h_{1}\right)}{\ell_{2}}\right)}{l_{2}} .
\end{aligned}
$$


Here, $A_{g 2}, A_{g s}, A_{2 s}$ are the Hamaker coefficients describing the long-range molecular repulsion or attraction, depending on the sign of a coefficient (see Table I), between the liquid-liquid and liquid-gas interface, solid-liquid and liquid-gas interface, and solid-liquid and liquid-liquid interface, respectively. $S_{1,2}<0$ are the spreading coefficients, describing the short-range molecular repulsion between the solid-liquid and liquid-liquid interface, and liquid-liquid and liquid-gas interface, respectively. $\ell_{1,2}$ are the equilibrium cutoff distances. The short-range repulsion between the solid-liquid and liquid-gas interface is not included. Note that (i) we include gravity terms in the pressures, since it has been shown that these terms may be important in a certain range of parameters [28,34], and (ii) we do not include the electronic component in the disjoining pressures [60], since for a single layer as well as for a bilayer system the inclusion of the electronic component does not result in the noticable improvement in matching of the theoretical predictions to the experiment, such as, for instance, the prediction of the critical film thickness at which the transition from bicontinuous structures to holes takes place [13,61].

The surface tension gradients, $\partial_{x} \sigma_{1,2}$ in Eqs. (2.1), (2.2) are caused by the gradients of the temperature at the liquid-liquid and the liquid-gas interface, respectively. We assume linear dependence of the surface tensions on temperature:

$$
\begin{array}{ll}
\sigma_{1}=\sigma_{1}^{(m)}-\gamma_{1}\left(T_{1}\left(z=h_{1}\right)-T_{1}^{(m)}\right), & \gamma_{1}>0, T_{1}\left(z=h_{1}\right)>T_{1}^{(m)}, \\
\sigma_{2}=\sigma_{2}^{(m)}-\gamma_{2}\left(T_{2}\left(z=h_{2}\right)-T_{2}^{(m)}\right), & \gamma_{2}>0, T_{2}\left(z=h_{2}\right)>T_{2}^{(m)},
\end{array}
$$

where $\sigma_{1,2}^{(m)}$ are the surface tensions at the corresponding melting temperatures $T_{1,2}^{(m)}$. Since the temperatures $T_{1,2}$ will be shown to depend on the thicknesses of the layers and also explicitly on the longitudinal cooordinate $x$ (in the interference irradiation mode only), the surface tension gradients are written as

$$
\begin{aligned}
& \partial_{x} \sigma_{1}=-\gamma_{1}\left(\left.\left(\partial_{h_{1}} T_{1}\right)\right|_{z=h_{1}} \partial_{x} h_{1}+\left.\left(\partial_{h_{2}} T_{1}\right)\right|_{z=h_{1}} \partial_{x} h_{2}+\left.\left(\partial_{x} T_{1}\right)\right|_{z=h_{1}}\right), \\
& \partial_{x} \sigma_{2}=-\gamma_{2}\left(\left.\left(\partial_{h_{1}} T_{2}\right)\right|_{z=h_{2}} \partial_{x} h_{1}+\left.\left(\partial_{h_{2}} T_{2}\right)\right|_{z=h_{2}} \partial_{x} h_{2}+\left.\left(\partial_{x} T_{2}\right)\right|_{z=h_{2}}\right) .
\end{aligned}
$$

In order to close the equation set, we need the specification of the thermal problem. This is discussed in the next section.

\subsection{Thermal problem}

The steady-state, long-wave energy equations in the two metallic layers and in the thin, purely conductive substrate are $[8,56]$

$$
\begin{aligned}
\frac{\kappa_{1,2}}{\rho_{1,2} C_{e f f}} \partial_{z z} T_{1,2}+\frac{\delta_{2} J}{\rho_{1,2} C_{e f f}} f(x)\left(1-R\left(h_{1}, h_{2}\right)\right) \exp \left(\delta_{1,2}\left(z-h_{2}\right)\right) & =0, \\
\frac{\kappa_{s}}{\rho_{s} C_{e f f}} \partial_{z z} T_{s} & =0,
\end{aligned}
$$

where $\kappa_{1,2, s}$ are the thermal conductivities, $C_{\text {eff }}$ is the effective heat capacity, $\delta_{1,2}$ are the optical absorption coefficients, $\delta_{2} J$ is the rate of the laser energy flux volumetric absorption into the upper layer, $\rho_{s}$ is the substrate density, and $R\left(h_{1}, h_{2}\right)$ is the dimensionless effective reflectivity function, which has been calculated [62] using the method decribed in detail in Ref. [63]. Short of presenting here the full reflectivity function, which is very cumbersome, the next equation shows the polynomial fit to the cross-section at $h_{1}=5 \mathrm{~nm}:$

$$
\begin{gathered}
h \leq 2 \times 10^{8} \mathrm{~m}: \\
R(h)=0.033-1.62 \times 10^{7} h+0.32 \times 10^{16} h^{2}-0.14 \times 10^{24} h^{3}+0.24 \times 10^{31} h^{4}, \\
h \geq 2 \times 10^{8} \mathrm{~m}: \\
R(h)=-0.31+0.41 \times 10^{8} h-0.98 \times 10^{15} h^{2}+0.97 \times 10^{22} h^{3}-0.34 \times 10^{29} h^{4},
\end{gathered}
$$




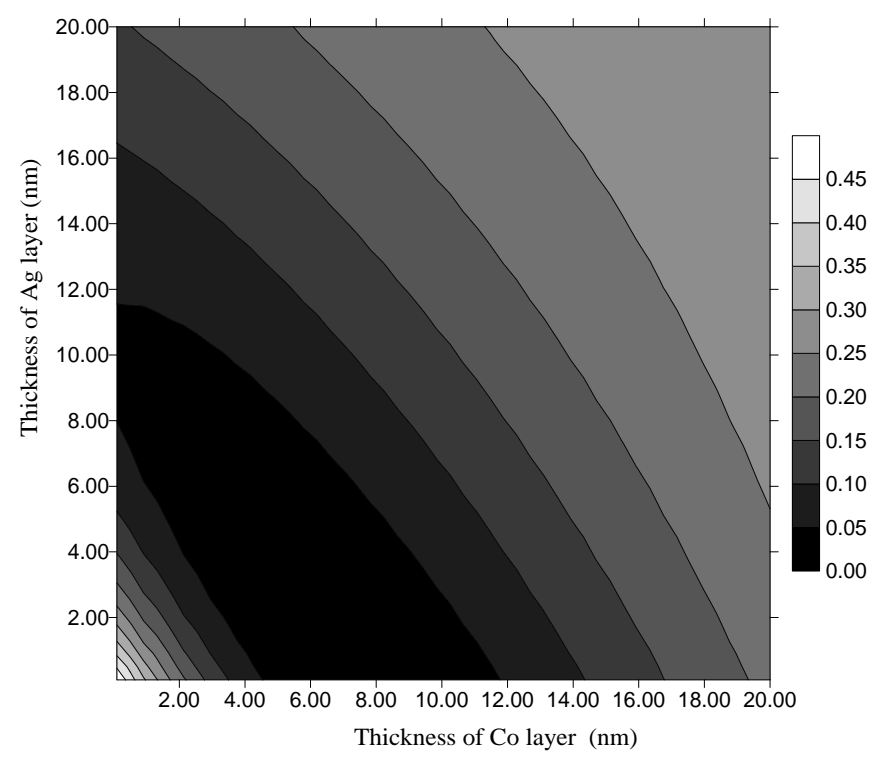

Figure 2. Contour plot of the reflectivity for $\mathrm{Ag} / \mathrm{Co} / \mathrm{SiO}_{2} / \mathrm{Si}$ system. The increased reflectivity when approaching zero top film thickness is due to the reflective underlying Si layer.

where $h$ denotes the thickness of the upper $(\mathrm{Ag})$ layer. This is plotted in Figure 5. Also, $f(x)$ is a function with the mean value one describing the irradiation intensity profile along the $x$-axis. We take

$$
f(x)=1+\epsilon \cos k_{i} x,
$$

where $0 \leq \epsilon \leq 1$ is the amplitude, and $k_{i}$ is the wavenumber. Nonzero values of $\epsilon$ stand for the twobeam interference mode of irradiation, while $\epsilon=0$ stands for irradiation by a single laser beam. (In the experiment, the wavenumber $k_{i}$ is uniquely related to the wavelength, $\lambda_{b}$ of the primary beam and the angle, $\theta_{i}$ between the two interfering beams, one of which has been splitted from the primary beam, through $2 \pi / k_{i}=\lambda_{b} / 2 \sin \left(\theta_{i} / 2\right)$.)

Eq. (2.5) is the Beer-Lambert law for the bilayer.

We also remark that the lateral dimension of the system is typically of the order of $1 \mathrm{~mm}$, which justifies the lubrication approximation in the substrate [51], Eq. (2.6) - despite that the substrate thickness $h_{s}$ is typically two orders of magnitude larger than the bilayer thickness $h_{20}$.

Figure 2 shows the contour plot of the reflectivity. The non-monotonic dependence of the reflectivity on thickness is evident, as is the increase of reflectivity as the thickness of the top film approaches zero. This is the result of increased reflectivity from the underlying reflective Si layer.

The long-wave boundary conditions for the set (2.5), (2.6) are

$$
\begin{gathered}
z=h_{2}: \quad \kappa_{2} \partial_{z} T_{2}=-\alpha\left(T_{2}-\Theta_{a}\right), \\
z=h_{1}: \quad \kappa_{1} \partial_{z} T_{1}=\kappa_{2} \partial_{z} T_{2}, T_{1}=T_{2}, \\
z=0: \quad \kappa_{1} \partial_{z} T_{1}=\kappa_{s} \partial_{z} T_{s}, T_{1}=T_{s}, \\
z=-h_{s}: \quad T_{s}=\Theta_{s},
\end{gathered}
$$

where $\Theta_{a}$ and $\Theta_{s}$ are the gas and substrate temperatures, respectively, and $\alpha$ is the heat transfer coefficient at the liquid-gas interface. 
The solution of this boundary-value problem in the liquid layers has the form

$$
\begin{aligned}
& T_{1}=-\frac{\delta_{2} J\left(1-R\left(h_{1}, h_{2}\right)\right)}{\delta_{1}^{2} \kappa_{1}} f(x) \exp \left(\delta_{1}\left(z-h_{2}\right)\right)+c_{11} z+c_{12}, \\
& T_{2}=-\frac{J\left(1-R\left(h_{1}, h_{2}\right)\right)}{\delta_{2} \kappa_{2}} f(x) \exp \left(\delta_{2}\left(z-h_{2}\right)\right)+c_{21} z+c_{22},
\end{aligned}
$$

where $c_{i j}$ are the complicated functions of $h_{1,2, s}, \kappa_{1,2, s}, \delta_{1,2}, J, \alpha, \Theta_{a}, \Theta_{s}$, which we obtained using the computer algebra system (CAS) (Mathematica). In the substrate, the temperature is an increasing linear function of $z$, as is evident from Eq. (2.6).

In the above presented formulation the heat conduction in the thick Si support layer is disregarded, and the temperature at the $\mathrm{SiO}_{2} / \mathrm{Si}$ interface is set equal to the mean substrate temperature $\Theta_{s}$, estimated from the transient heating model that incorporates phase change [62] for periods when the system is in the liquid state and for the typical values of the laser energy and the bilayer film thickness employed in this study. $\Theta_{s}$ is obtained by averaging over many pulses the temperature rise and fall within the width of the Gaussian pulse. Practically, this formulation also makes it possible to evaluate at every time step the steady-state temperatures of the metal layers and the derivatives of these temperatures with respect to thicknesses $h_{1}$ and $h_{2}$. Note also, that even without accounting for the heat conduction in the support layer, this layer still exerts the important influence on the system's steady-state temperature field, since the effective reflectivity $R$ is calculated for the full system metal $1 /$ metal $_{2} / \mathrm{SiO}_{2} / \mathrm{Si}$. The computed temperature profiles are not shown here, since they are presented in Ref. [58].

The transient heating model [62] allows to fit the dependence of $\Theta_{s}$ on $h_{20}$ for a given rate of change of the thermal flux, $J$. For the $J$ value stated in the next section, that corresponds to the laser energy density $100 \mathrm{~mJ} / \mathrm{cm}^{2}$, the fit is:

$$
\begin{gathered}
7 \mathrm{~nm} \leq h_{20} \leq 13 \mathrm{~nm}: \\
\Theta_{s}=109452.2-64210.4 h_{20}+14715.7 h_{20}^{2}-1626 h_{20}^{3}+87 h_{20}^{4}-1.8 h_{20}^{5}, \\
13 \mathrm{~nm} \leq h_{20} \leq 17 \mathrm{~nm}: \\
\Theta_{s}=2126.1+3.93 h_{20}+0.786 h_{20}^{2},
\end{gathered}
$$

This is shown in Figure 3.

For $\epsilon=0$ (which entails $\left.\left(\partial_{x} T_{1,2}\right)\right|_{z=h_{1,2}}=0$ in Eqs. (2.3) and (2.4)), we found that the thermocapillary surface tension gradient terms $-\partial_{x} \Phi_{i j} \partial_{x} \sigma_{k}$ in the evolution equations (2.1) and (2.2) are negative (stabilizing) in the thin $\mathrm{Ag} / \mathrm{Co}$ (and $\mathrm{Co} / \mathrm{Ag}$ ) films, and positive (destabilizing) in the thick films. For $\mathrm{Ag} / \mathrm{Co}$ films, this transition happens at $h_{20} \sim 30 \mathrm{~nm}$. Figure 4(a) shows the comparison of the linearized terms at the RHS of the evolution equation (2.2). These terms are calculated at $h_{10}=5 \mathrm{~nm}, h_{20}=35$ $\mathrm{nm}$ and at the amplitudes of the interfaces deformations that are set equal to $0.1 \mathrm{~nm}$, the values that are within reach of the linear approximation. (Deformation $=$ amplitude $\times \cos k x$.) Figure 4(b) shows the temperature gradients $\partial T / \partial h$; the transition from a positive (stabilizing) to a negative (destabilizing) gradient can be clearly seen at $h_{20}=32 \mathrm{~nm}$.

This transition is similar to the case of a single-layer metal film; in Ref. [8] it is shown that Co films on the $\mathrm{SiO}_{2}$ substrate are destabilized by the thermocapillary forces at thicknesses larger than roughly $10 \mathrm{~nm}$. For bilayers of $\mathrm{Ag} / \mathrm{Co}$ on $\mathrm{SiO}_{2} / \mathrm{Si}$ the transition does not occur at such a small thickness likely due to the reflectivity of the $\mathrm{Si}$, which results in the distinctly different reflectivity curve of the system for small thicknesses, see Figure 5. Overall, the reason for thermocapillary destabilization is the temperature response to the interplay of the thickness-dependent absorption and reflectivity. Averaging Eqs. (2.11) and (2.12) from $z=0$ to $z=h_{2}$, one obtains that the dominant part of the averaged temperature is proportional to $\left(1-R\left(h_{1}, h_{2}\right)\right)\left(1-\exp \left(-\delta_{i} h_{2}\right)\right)$ (see also Eq. (9) in Ref. [8] and its discussion on pp. 6 and 7 there, for the single layer case). For the typical value $\delta \sim 10^{8} \mathrm{~m}^{-1}$ and the reflectivity approximated by Eqs. (2.7) and (2.8) this is plotted in the inset of Figure 5. The transition at $h_{20} \sim 30$ nm roughly 


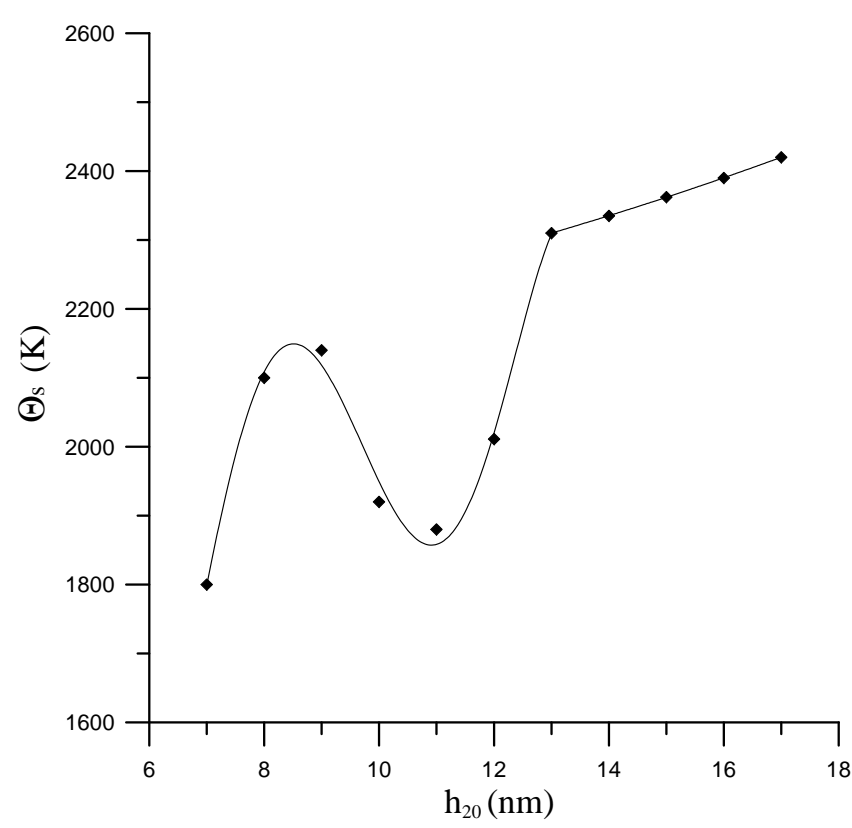

Figure 3. Substrate temperature $\Theta_{s}$ vs. bilayer thickness $h_{20}$ (fit to the output of the transient heating model of Ref. [62]).

(a)

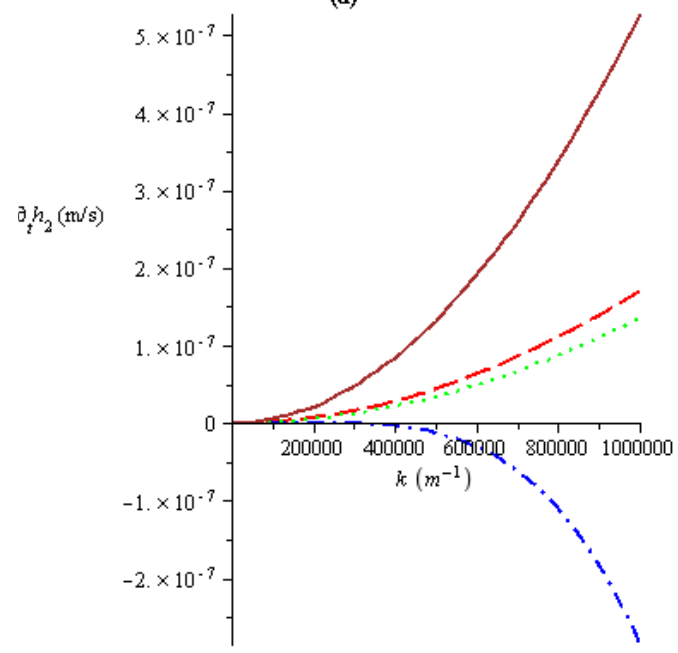

(b)

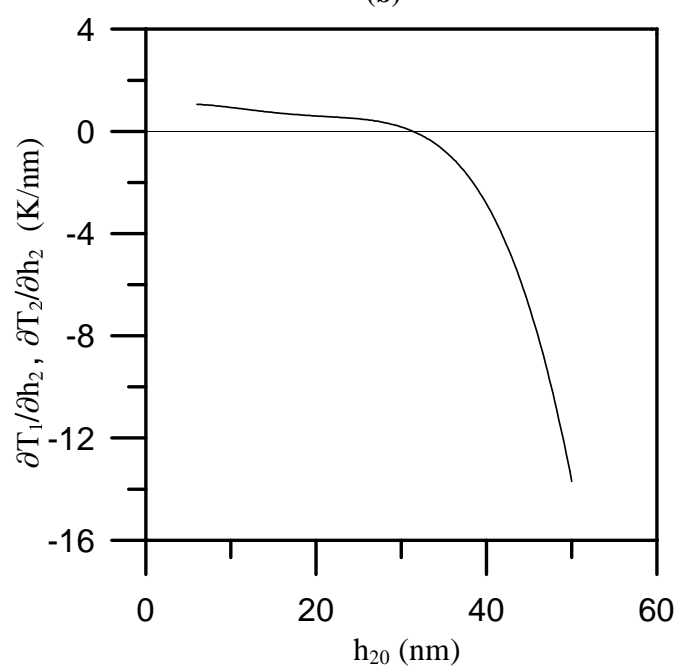

Figure 4. (Color online). (a) The linearized terms at the RHS of Eq. (2.2) at the interfacial deformations with the amplitude $0.1 \mathrm{~nm}$ from the mean values $h_{10}=5 \mathrm{~nm}$, $h_{20}=35 \mathrm{~nm}$. Dashed line: 1st term; dash-dotted line: 2nd term; dotted line: 3rd term; solid line: 4th term. Values along the vertical axis show the contribution of each term to the total velocity of the interface, not the total velocity itself. (b) Dominant temperature gradients in Eqs. (2.3) and (2.4) vs. $h_{20}$. $\left(\partial T_{1} / \partial h_{2}\right.$ is very close to $\partial T_{2} / \partial h_{2}$ on the scale of the figure, thus one line is shown for both; the other two gradients are much smaller and thus not shown.) 


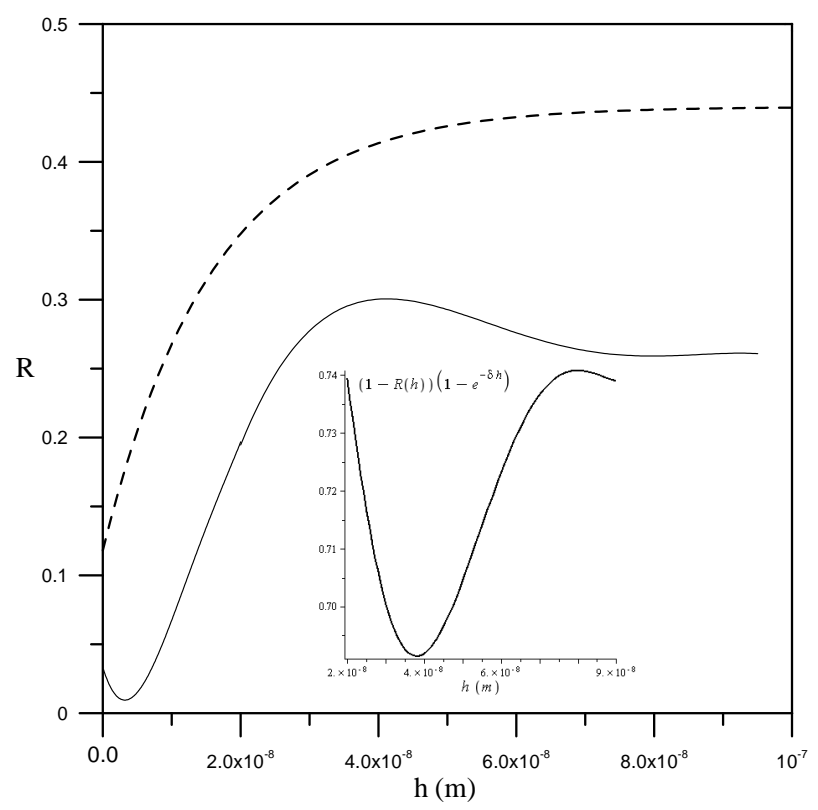

Figure 5. Solid line: Reflectivity $R$ of $\mathrm{Ag} / \mathrm{Co} / \mathrm{SiO}_{2} / \mathrm{Si}$ system as a function of the thickness of the Ag layer, here denoted by $h$; the thickness of the bottom Co layer is 5 nm. Dashed line: Reflectivity $R$ of $\mathrm{Co} / \mathrm{SiO}_{2}$ system as a function of the excess thickness (over $5 \mathrm{~nm}$ ), $h$, of the Co layer. This $R(h)=r_{0}\left(1-\exp \left(-a_{r}\left(h+5 \times 10^{-9}\right)\right)\right)$, where $r_{0}=0.44$ and $a_{r}^{-1}=16 \times 10^{-9} \mathrm{~m}$ (from Ref. [8]). Inset shows the characteristic product for the $\mathrm{Ag} / \mathrm{Co} / \mathrm{SiO}_{2} / \mathrm{Si}$ system.

coincides with the maximum of the reflectivity curve and with the minimum of the product stated above.

Destabilization by thermocapillarity in thick $\mathrm{Ag} / \mathrm{Co}$ films is manifested in the appearance of the secondary maximum on the linear dispersion curve at small wavenumbers. With the increase of $h_{20}$, this maximum grows fast and soon the associated wavelength prevails. We call this effect the exchange of stability for the lack of a better term (not to confuse with the principle of exchange of stabilities, see for instance Ref. [64]). When stability is exchanged, the wavelength jumps to a larger value. Thus this kind of destablization leads to a sudden increase of particle spacing upon dewetting. We do not further investigate this effect here, since for thick films there is yet no data from experiment to compare the model predictions to.

For the parameters stated in the next section, and for $\lambda_{i}=2 \pi / k_{i}=347 \mathrm{~nm}$ (which corresponds to $\lambda_{b}=266 \mathrm{~nm}$ and $\left.\theta_{i}=45^{\circ}\right)$, the in-plane thermal gradients $|\Delta T| / \lambda_{i}$ are estimated from the steady-state thermal model presented above to be of the order of $0.05 \mathrm{~K} / \mathrm{nm}$. This is comparable to the estimate of the vertical thermal gradient $(\sim 0.1 \mathrm{~K} / \mathrm{nm})$. However, the transient heat conduction model $[8,62]$ predicts vertical thermal gradients ranging from 0.5 to $0.7 \mathrm{~K} / \mathrm{nm}$ in the liquid phase. For this reason, we omit the $\left.\left(\partial_{x} T_{1,2}\right)\right|_{z=h_{1,2}}$ terms in Eqs. (2.3) and (2.4).

\subsection{Parameters}

The typical values of the physical parameters for the $\mathrm{Ag} / \mathrm{Co} / \mathrm{SiO}_{2} / \mathrm{Si}$ system are as follows: $\eta_{1}=4.46 \times$ $10^{-3} \mathrm{~Pa}-\mathrm{s}, \eta_{2}=3.88 \times 10^{-3} \mathrm{~Pa}-\mathrm{s}, \sigma_{1}^{(m)}=0.168 \mathrm{~J} / \mathrm{m}^{2}, \sigma_{2}^{(m)}=0.925 \mathrm{~J} / \mathrm{m}^{2}, \gamma_{1}=\gamma_{2}=5 \times 10^{-6}-5 \times 10^{-4}$ $\mathrm{J} /\left(\mathrm{K} \mathrm{m}^{2}\right), \rho_{1}=7.75 \times 10^{3} \mathrm{Kg} / \mathrm{m}^{3}, \rho_{2}=9.32 \times 10^{3} \mathrm{Kg} / \mathrm{m}^{3}, T_{1}^{(m)}=1768 \mathrm{~K}, T_{2}^{(m)}=1235 \mathrm{~K}, \Theta_{a}=300$ $\mathrm{K}, \delta_{1}=\delta_{2}=10^{4}-10^{8} \mathrm{~m}^{-1}, \alpha=10^{3} \mathrm{~W} /\left(\mathrm{K} \mathrm{m}^{2}\right), \kappa_{1}=34 \mathrm{~W} /(\mathrm{K} \mathrm{m}), \kappa_{2}=185 \mathrm{~W} /(\mathrm{K} \mathrm{m}), \kappa_{s}=50$ 


\begin{tabular}{ccc}
\hline \hline Hamaker const. & Set I & Set II \\
\hline$A_{g 2}$ & $-10^{-20} \mathrm{~J}$ & $-10^{-19} \mathrm{~J}$ \\
$A_{g s}$ & $4 \times 10^{-20} \mathrm{~J}$ & $4 \times 10^{-20} \mathrm{~J}$ \\
$A_{2 s}$ & $4 \times 10^{-21} \mathrm{~J}$ & $2 \times 10^{-20} \mathrm{~J}$ \\
\hline \hline
\end{tabular}

TABLE 1. Hamaker coefficients for the $\mathrm{Ag} / \mathrm{Co} / \mathrm{SiO}_{2} / \mathrm{Si}$ system. The positive sign corresponds to destabilizing (attractive) interaction between interfaces.

$\mathrm{W} /(\mathrm{K} \mathrm{m}), h_{s}=400 \mathrm{~nm}$, and $J=1.25 \times 10^{11} \mathrm{~J} /\left(\mathrm{s} \mathrm{m}^{2}\right)$. Values of $\gamma_{1,2}$ and $\delta_{1,2}$ are somewhat uncertain, thus the possible range of values is stated. To the best of our knowledge, the magnitudes of the Hamaker coefficients for the metallic systems like $\mathrm{Ag} / \mathrm{Co} / \mathrm{SiO}_{2} / \mathrm{Si}$ are not known with precision and vary based on the type of calculation used to evaluate them, as noted in Ref. [16]. Generally, values of the Hamaker coefficients may vary from $10^{-22} \mathrm{~J}$ to $10^{-18} \mathrm{~J}[65,66]$. Here we have utilized the two sets of coeffcients used in Ref. [16] which successfully bounded the experimental values based on the energy rate theory. The sets are shown in the Table I.

For the ease of comparison with the results of the experiments and in order to facilitate communications within our interdisciplinary collaboration, we kept all equations in the dimensional form.

\section{Linear stability analysis}

Assuming $\epsilon=0$, the standard linear stability analysis (LSA) of the problem (2.1) - (2.4), (2.11), (2.12) results in the quadratic dispersion relation

$$
\omega^{2}(k)-\left(a_{11}+a_{22}\right) \omega(k)+a_{11} a_{22}-a_{12} a_{21}=0,
$$

where $\omega(k)$ is the growth rate of a perturbation, $k$ is the perturbation wavenumber, and the coefficients $a_{i j}$ are the complicated functions (determined again with the help of the CAS) of $k, h_{10,20}$, the Hamaker coefficients, and other physical parameters.

Eq. (3.1) may have two real and distinct solutions, one real solution, or two complex conjugate solutions. For the parameters of interest, only the first situation was ever found. In other words, only the monotonous modes of instablity were detected. Also, the first solution curve $\omega_{1}(k)$ has a typical longwave shape, and the second solution $\omega_{2}(k)$ is negative. Thus for a thin Ag/Co film the situation is much simpler than the general case, where the two growth rates may be of the same sign and commensurable, and either one or both may exhibit multiple maximas $[26,27,69]$. Besides the thick $\mathrm{Ag} / \mathrm{Co}$ film case mentioned in Section 2.2, another example of such more complicated situation, i.e. (i) $\omega_{1}>0$ for $k<k_{c 1}$ and features one maximum or two maximas depending on $h_{20}$, (ii) $\omega_{2}>0$ for $k<k_{c 2}$, and (iii) $\omega_{1} \gg \omega_{2}$ for $k<\max \left(k_{c 1}, k_{c 2}\right)$ has been encountered in our modeling of the $\mathrm{Co} / \mathrm{Ag} / \mathrm{SiO}_{2} / \mathrm{Si}$ system [58], which is not considered in this paper.

Before presenting the LSA results for the $\mathrm{Ag} / \mathrm{Co} / \mathrm{SiO}_{2} / \mathrm{Si}$ system, we show in Figure 6 the comparison of the calculated particle spacing, $\lambda\left(=2 \pi / k_{\max }\right.$, where $k_{\max }$ corresponds to the maximum point on the longwave dispersion curve) to one obtained in the experiment on the single-layer film $(\mathrm{Co} / \mathrm{SiO})_{2}$. To obtain the dispersion curve for the latter system, the upper layer $(\mathrm{Ag})$ is eliminated from the governing system of equations and the boundary conditions for the temperature. The convective boundary condition (2.9) (where the subscript 2 is eliminated) is now prescribed on the Co/air interface, and the fixed temperature condition (2.10) is still prescribed at the bottom of the $\mathrm{SiO}_{2}$ substrate. The material parameters of Co are as follows: $\eta=4.46 \times 10^{-3} \mathrm{~Pa}-\mathrm{s}, \sigma^{(m)}=1.88 \mathrm{~J} / \mathrm{m}^{2}, \gamma=5 \times 10^{-5} \mathrm{~J} /\left(\mathrm{K} \mathrm{m}^{2}\right)$, $\rho=9.32 \times 10^{3} \mathrm{Kg} / \mathrm{m}^{3}, T^{(m)}=1768 \mathrm{~K}, \delta=10^{6} \mathrm{~m}^{-1}$, and $\kappa=34 \mathrm{~W} /(\mathrm{K} \mathrm{m})$; other parameters $\left(\Theta_{a}, \alpha, \kappa_{s}\right.$, $\left.h_{s}, J\right)$ have values stated above. Also, the expression for reflectivity is shown in the caption to Figure 5 , and the chosen value of a single Hamaker coefficient is $10^{-19} \mathrm{~J}$. Due to the lack of data of the type Eqs. (2.13), (2.14) for the thin Co film $(0 \leq h \leq 10 \mathrm{~nm})$, we chose to fix the substrate temperature $\Theta_{s}$ to 1850 $\mathrm{K}$, which is slightly larger than the Co melting temperature and the correct order of magnitude [8]. 


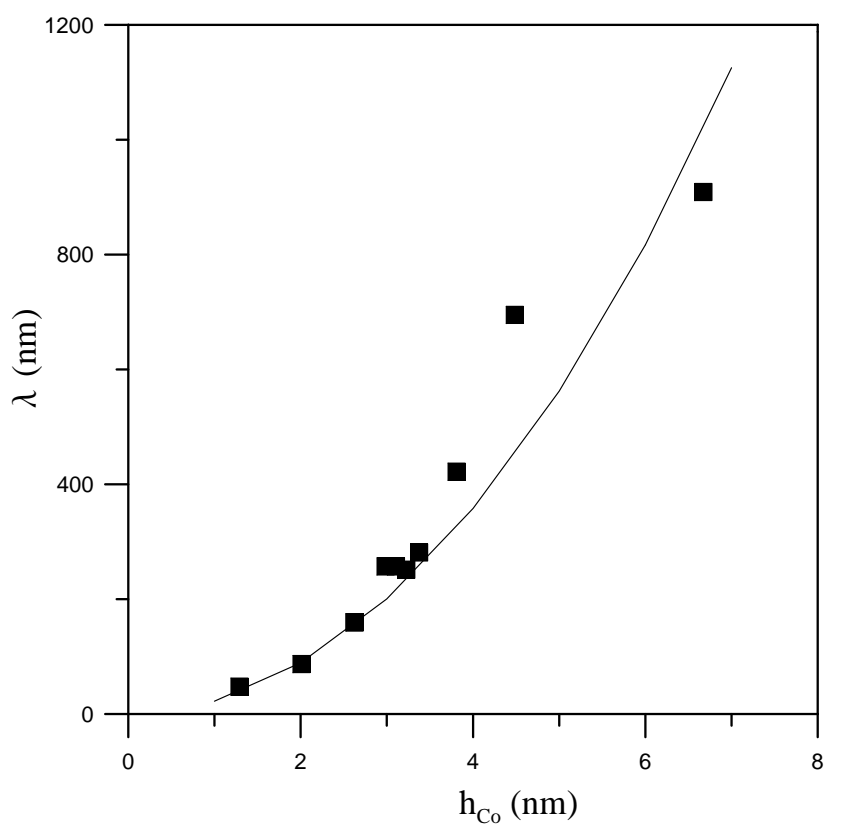

Figure 6. Particle spacing from the LSA (solid line) and from experiment (solid squares), for a thin Co film. Solid line is perfectly fitted by a quadratic, $\lambda \sim 24 h_{\text {Co }}^{2}$. Similar quadratic dependence on thickness (up to a coefficient) has been determined in Ref. [8]

Turning now to bilayers, Figure 7 shows the comparison of the particle spacing from LSA to data from experiment. Here the Co layer thickness is fixed at $5 \mathrm{~nm}$, and the Ag layer thickness is varied. LSA data is presented for two sets of Hamaker coefficients and, for each set, both the constant $\Theta_{s}$ approximation $(1850 \mathrm{~K})$ and the fitted one (Eqs. (2.13), (2.14) and Figure 3) are shown. The first set of coefficients provides better match, and within this set, the line (c) fits data better in the least squares sense than the line (a).

\section{Computations of the bilayer dewetting and morphology evolution}

In this section we present the results of computations of the $\mathrm{Ag} / \mathrm{Co}$ bilayer dynamics when the interference of the laser beams is activated. We take $\epsilon=0.99, k_{i}=k_{\max } / 2$ and the domain length $L=4\left(2 \pi / k_{i}\right)$. Periodic boundary conditions are imposed at $x=0$ and $x=L$. Thus in the absence of interference $(\epsilon=0)$ the number of particles in $L$, as expected from LSA, is eight (before coarsening), and interference is expected to affect the particle formation and perhaps the particle number.

Evolution equations (2.1), (2.2) are solved numerically using the method of lines. Integration in time is performed using the stiff ODE solvers RADAU [67] (implements a class of implicit Runge-Kutta methods with automatic order switching) and/or VODE [68] (implements a class of backward differencing methods with automatic order switching), whereas the discretization in space is carried out in the conservative form using the second order finite differencing with half-node interpolation, on a spatially uniform grid. VODE is more efficient, however it is less stable in the initial phase of integration when the shapes of the interfaces are irregular (i.e., pseudo-random). Thus we usually employ a combination strategy, where the integration is carried out by RADAU until the shapes coarsened sufficiently (judged by an easily computed first moment of an autocorrelation function), and then switch to VODE. 


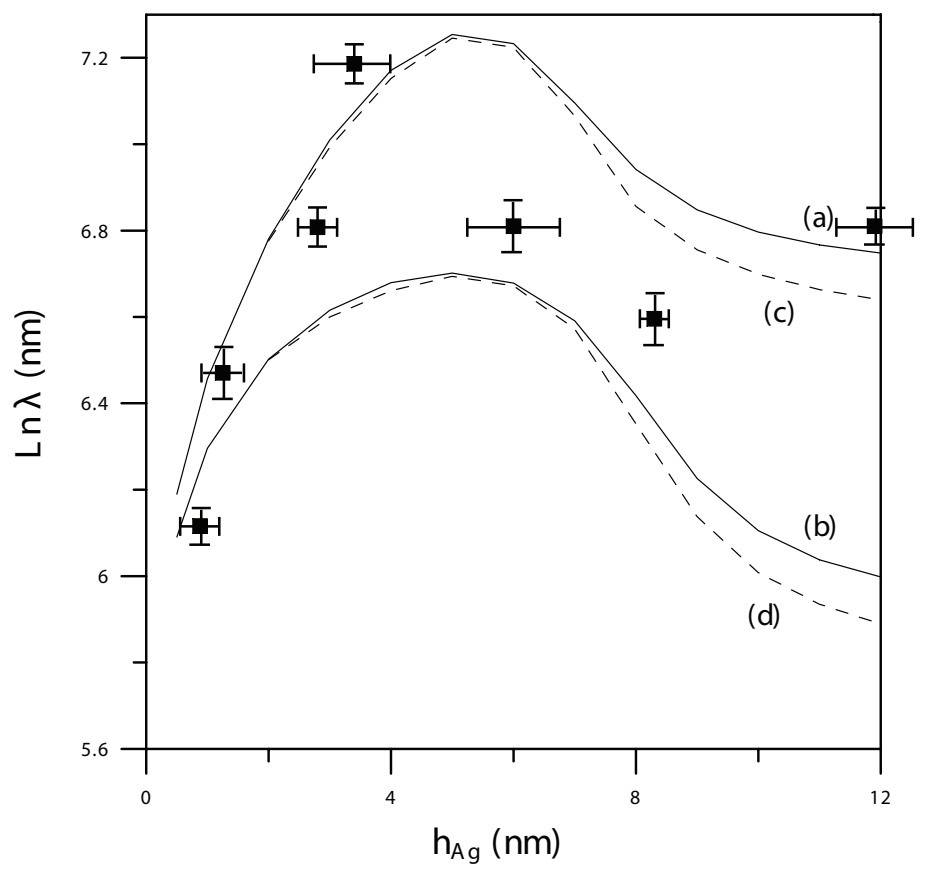

Figure 7. Particle spacing from the LSA and from experiment (solid squares), for the $\mathrm{Ag} / \mathrm{Co}$ bilayer. Lines (a) and (c): Hamaker coefficients Set I (Table I). Lines (b) and (d): Hamaker coefficients Set II. Solid lines: $\Theta_{s}=1850 \mathrm{~K}$; dashed lines: $\Theta_{s}$ from the fit (2.13), (2.14). For all curves $\gamma_{1}=\gamma_{2}=5 \times 10^{-5}, \delta_{1}=\delta_{2}=10^{6}$. Decreasing (increasing) $\delta_{1,2}$ within the limits shown in Sec. 2.3 shifts the dispersion curve up (down) by at most 0.2 units on the logarithmic scale; decreasing or increasing $\gamma_{1,2}$ has similar effect.

For computations, we use the first set of the Hamaker coefficients, $\Theta_{s}=1850 \mathrm{~K}$ and $\delta_{1,2}, \gamma_{1,2}$ as shown in the caption to the Figure 7. To avoid films rupture at the substrate the spreading coefficients are set to non-zero negative values $S_{1}=-0.9 \mathrm{~J} / \mathrm{m}^{2}, S_{2}=-0.3 \mathrm{~J} / \mathrm{m}^{2}$; the cut-off lengths are $\ell_{1,2}=0.2 \mathrm{~nm}$.

In all cases, the layers evolve in the bending mode (see Figure 1) at all times. This is confirmed using the criteria applicable to the linear regime (small deformations) and to the nonlinear regime (large deformations), suggested respectively in Refs. [27] and [26].

As an easy-to-start-with example, Figure 8 shows the state of the morphology prior to coarsening, where the initial condition is not random, but periodic with the wavelength $2 \pi / k_{\max }$. As stated above, eight core-shell particles emerge upon dewetting. It can be seen that larger particles are formed at the peaks of deposited laser energy, i.e. where the interference is constructive. The reason for this is the stabilizing effect on the dynamics of the interfaces of the heat generation terms in the energy equations (2.5) $[49,50,56]$. These terms are maximal at the maximas of $f(x)$.

Next, we focus on the case of the initial conditions which are a small, uncorrelated, volume-preserving random perturbations of the mean thicknesses $h_{10,20}$ of the layers. We computed evolution with either $h_{10}$ fixed at $5 \mathrm{~nm}$ and $h_{20}$ ranging from $7 \mathrm{~nm}$ to $16 \mathrm{~nm}$, or with $h_{20}-h_{10}$ fixed at $5 \mathrm{~nm}$ (top layer thickness is $5 \mathrm{~nm}$ ), and $h_{10}$ ranging from $2 \mathrm{~nm}$ to $7 \mathrm{~nm}$. The effect of interference heating is easier to see in the first case.

Figure 9 shows the evolution of the morphology for a thin $(2 \mathrm{~nm})$ upper film (i.e., $h_{20}=7 \mathrm{~nm}$ ). Dewetting ceases at around $t=0.9 \times 10^{-3} \mathrm{~s}$ with the formation of six particles, the two of which have not separated and are still joined together (Figure 9(a)). For comparison, Figure 9(d) shows the morphology at this stage when the interference is turned off. At later time, these two particles re-arrange their shapes into a regular shapes, while the shapes and spacings of the other four particles do not change 


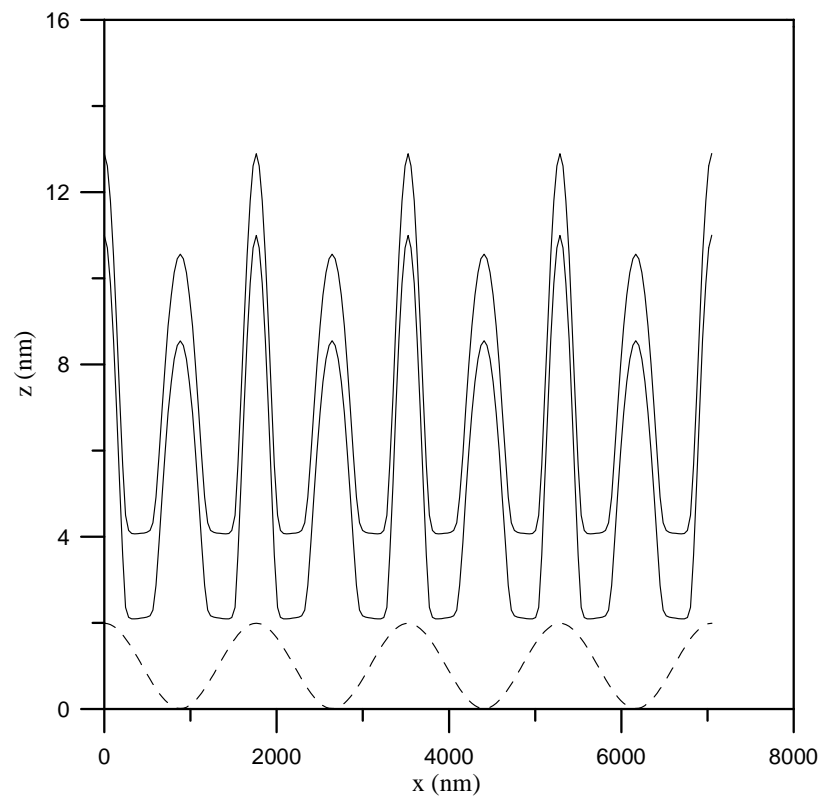

FiguRE 8. Core-shell nanoparticles (solid lines) formed upon dewetting of a sinusoidally perturbed bilayer with active laser beam interference. $h_{10}=5 \mathrm{~nm}, h_{20}=7 \mathrm{~nm}$. Dashed line: shape of the deposited laser energy, i.e. the function $f(x)=1+0.99 \cos k_{i} x$.

appreciably (Figure 9(b)). At last, six regular core-shell particles form. Then the coarsening stage begins where some particles grow at the expense of the other particles until a single large particle remains; some unusual features of this coarsening will be explained momentarily. Here, we emphasize that prior to this coarsening stage the number of formed particles is reduced from eight to six, compared to the evolution without the interference effect.

Next, Figure 10 shows the evolution of the morphology for a thicker upper film $\left(h_{20}=10 \mathrm{~nm}\right)$. Prior to the onset of particles coarsening, eight particles are formed from the bottom (Co) layer; dewetting of the top layer is incomplete, so that four two-hump particles are formed (Figure 10(a)). Apparently, the intereference is still stronly affecting the top layer, but its influence on the bottom layer at this stage is minimal. However, when coarsening starts, it is those particles in both layers located at the interference maximas that start to coarsen first (Figure 10(b)). This is again consistent with the finding that hotter areas, where more heat is generated, are more stable. After a particle or several particles are eliminated by coarsening, the larger coarsened particles are formed at the locations of interference maximas. This can be concluded from Figure 10(b), and is also explicitly shown in Figure 11, which is discussed next. The mechanism seems clear - since statistically larger particles are more likely to form at the hotter areas due to enhanced stability there, they grow in size at the expense of smaller particles located at colder areas; that coarsening proceeds by the flow of mass from smaller to larger particles is well known [70].

Figure 11 shows the evolution of the morphology for an even thicker upper film $\left(h_{20}=13 \mathrm{~nm}\right)$. Here, seven particles are formed from the bottom layer upon the completion of dewetting and prior to the onset of coarsening (Figure 11(a)), compared to eight particles when the interference is turned off (Figure 11(d)). These particles are embedded in the top layer, which deforms much less (the effect of weakening of the attactive potential). The deformation pattern of the top layer follows the bottom layer. Here the effect of interference seems to be the decrease of the number of particles from eight to seven. Figure 11(b) shows the advanced stage of coarsening when only five particles remain, and have just been formed, in the bottom layer. As has been stated above, their locations coincide quite accurately with interference maximas (a small compressive shift of the first and the fifth particle can be noticed). And Figure 11(c) 
(a)

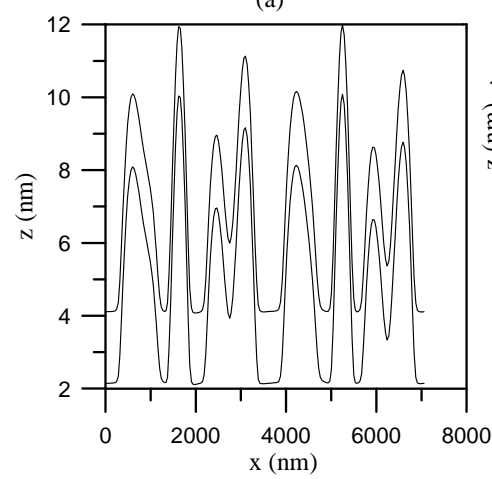

(c)

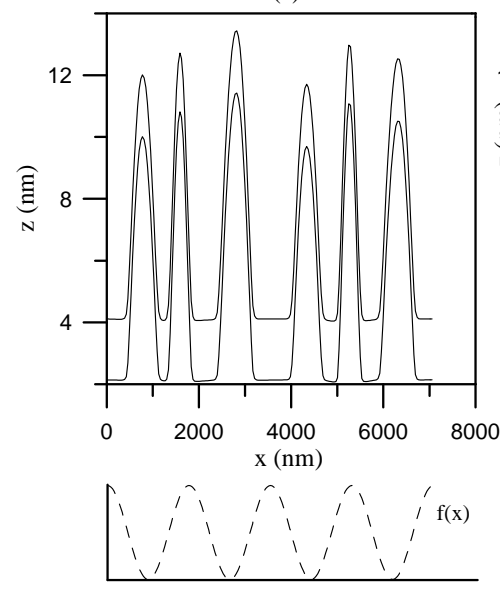

(b)

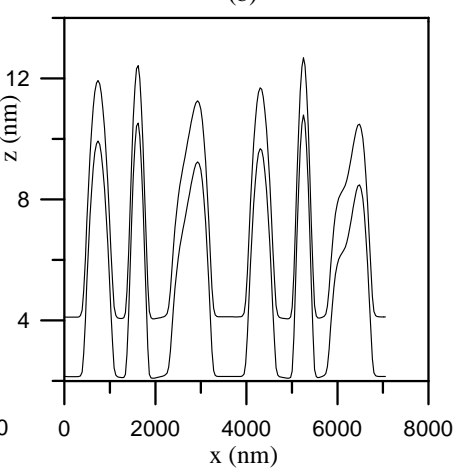

(d)

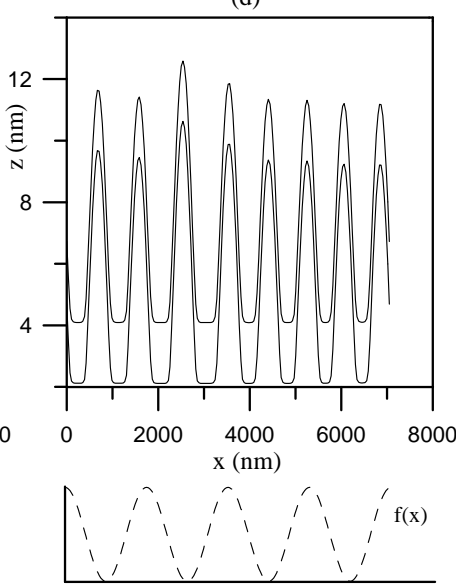

FiguRE 9. (a)-(c): Time frames of bilayer evolution with active laser beam interference towards core-shell nanoparticles. $h_{10}=5 \mathrm{~nm}, h_{20}=7 \mathrm{~nm}$. (a) $t=0.89 \times 10^{-3} \mathrm{~s}$, (b) $t=0.11 \times 10^{-2} \mathrm{~s},(\mathrm{c}) t=0.129 \times 10^{-2} \mathrm{~s} .(\mathrm{d})$ : Same as (a), but without inteference.

shows an even later stage - the four remaining particles, upon their formation, are again located at the interference maximas, this time with perfect precision.

Lastly, Figure 12 shows the evolution of the morphology for a thin bottom layer, $h_{10}=2 \mathrm{~nm}$, and thicker top layer $\left(h_{20}=7 \mathrm{~nm}\right)$. Somewhat similar to other snapshots, here seven particles are formed upon the bottom film dewetting, of which one, located at the interference maximim, does not complete dewetting (Figure 12(a)). A while later this double-hump particle coarsens into a large particle, bringing the total number of particles to six (Figure 12(b)). Then, the particles to the left and to the right of this large particle transfer their mass to the large particle (Figure 12(c,d)). We interrupted the computation shortly after the four-particle state has been reached. Although less obvious than the other computational results, this sequence of snapshots seems to demonstrate that again the particles at or close to the interference maximas have better chances of survival, i.e. the large particle is always at such location, as well as the particles at $x=0$ and $x=1100 \mathrm{~nm}$. The surface of the top layer is slowly responding to the shape changes of the interface between the layers; interference does not affect its evolution. 

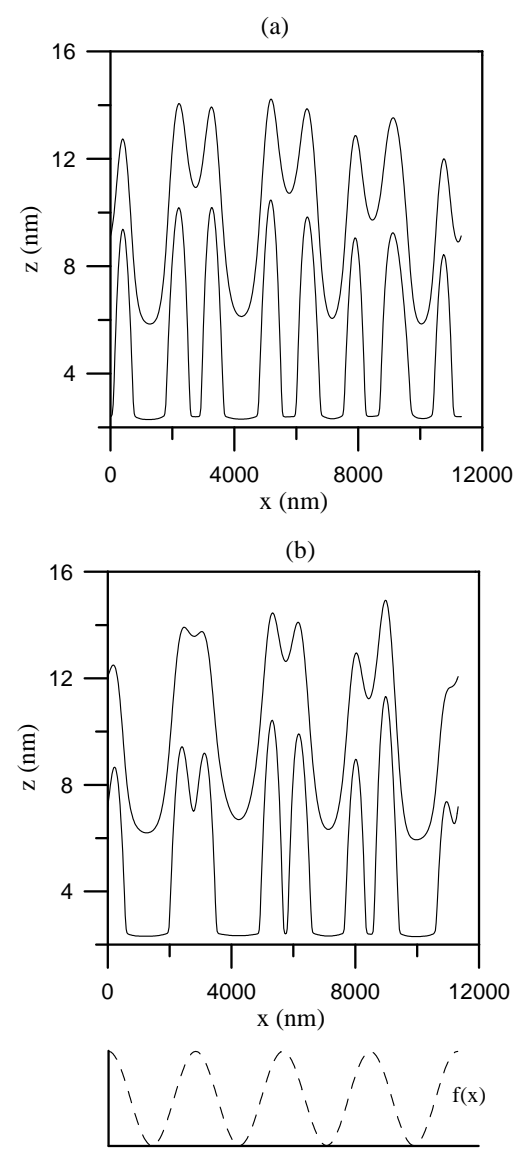

FIGURE 10. Time frames of bilayer evolution with active laser beam interference towards core-shell nanoparticles. $h_{10}=5 \mathrm{~nm}, h_{20}=10 \mathrm{~nm}$. (a) $t=0.56 \times 10^{-2} \mathrm{~s}$, (b) $t=$ $0.73 \times 10^{-2} \mathrm{~s}$.

\section{Conclusions}

In this paper, we studied the nanostructure-forming dynamics in pulsed-laser irradiated, metallic bilayer films, using the long-wave approximation. The full heat conduction problem, which includes the thickness dependent reflectivity and absorption in the bilayer, and also the interference heating effect is formulated, solved exactly, and the solution is coupled to the evolution equations for the thickness functions of the two layers. Intermolecular forces are accounted for in the evolution equations, and the thermocapillary (Marangoni) effect resulting from the thickness-dependent temperature is also taken into account at the gas-liquid and liquid-liquid interfaces. In the absence of the interference heating effect, the linear stability analysis provides the nanoparticle spacing as a function of a thickness of a top layer. This calculated spacing compares qualitatively well to one from the experiment. Results of simulations in the interference heating mode of the fully nonlinear evolution equations are presented. It is found that the interference heating affects both the number of formed particles and their positions along the substrate. In most simulations the number of particles is less than the number of one formed when interference is inactive, and the particles locations coincide with the interference maximas. With the increase of the top layer thickness, the deformation of the top layer becomess smaller, and the core-shell particle morphology is 
(a)

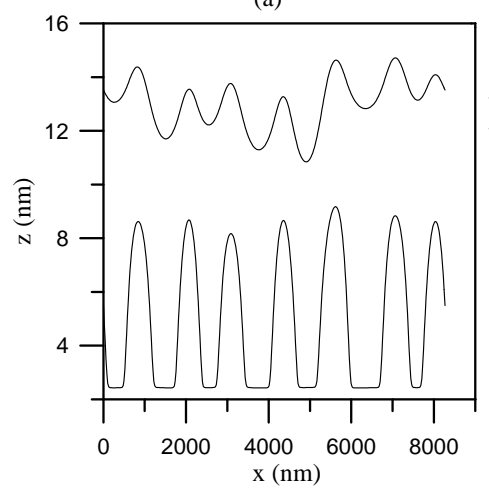

(c)

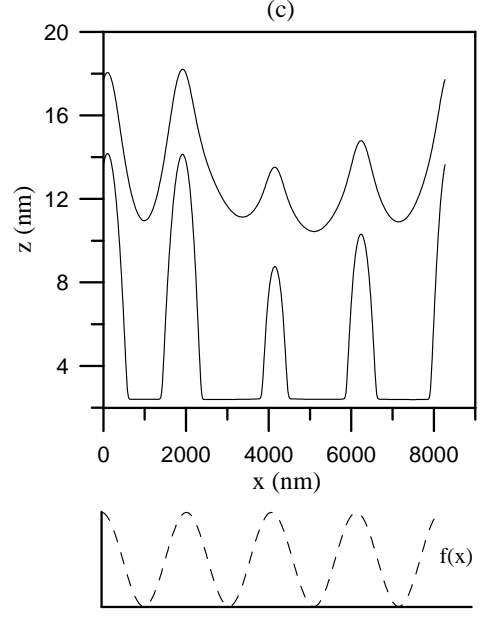

(b)

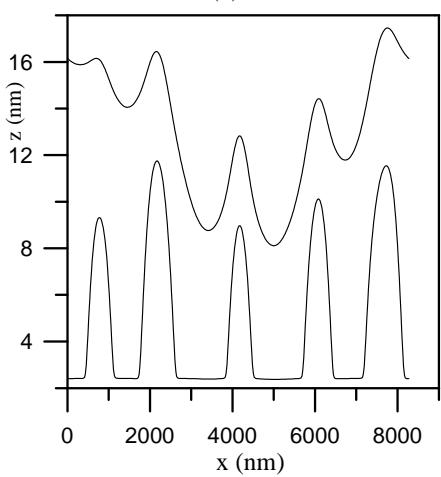

(d)

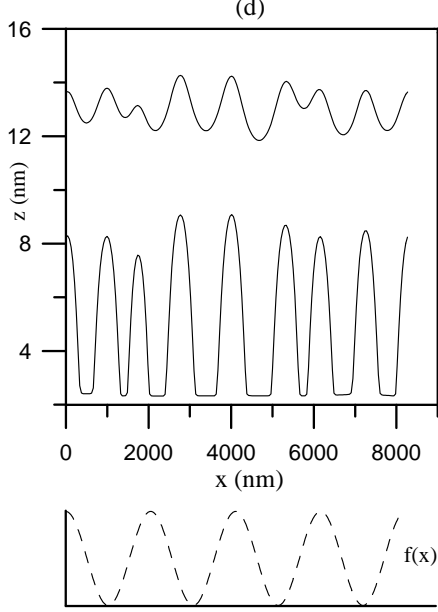

FIGURE 11. (a)-(c): Time frames of bilayer evolution with active laser beam interference towards embedded nanoparticles. $h_{10}=5 \mathrm{~nm}, h_{20}=13 \mathrm{~nm}$. (a) $t=0.59 \times 10^{-2} \mathrm{~s}$, (b) $t=0.13 \times 10^{-1} \mathrm{~s},(\mathrm{c}) t=0.31 \times 10^{-1} \mathrm{~s}$. (d): Same as (a), but without inteference.

replaced by the embedded ones, i.e. the particles of the bottom layer material in the matrix of the upper layer material. Still, the interference effect as described persists.

Acknowledgements. M.K. acknowledges the support of WKU Faculty Scholarship Council via grants 10-7016 and 10-7054. R.K. acknowledges the support of NSF grants CAREER DMI-0449258 and CMMI-0855949. R.K. would also like to thank Dr. Jeremy Strader for help with the bilayer optical reflectivity calculations. 
(a)

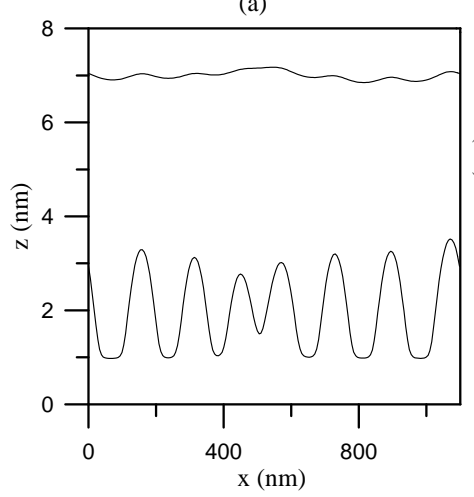

(c)

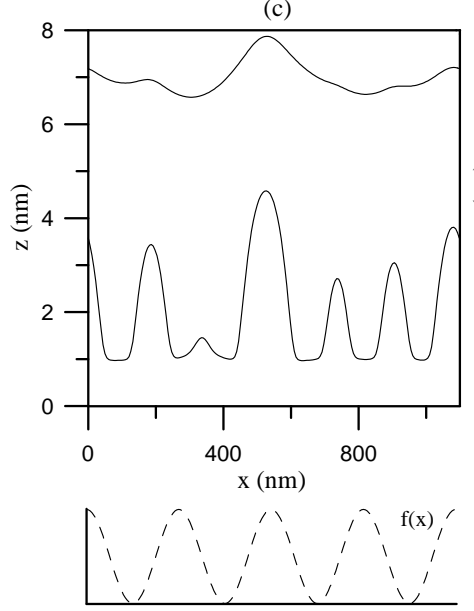

(b)

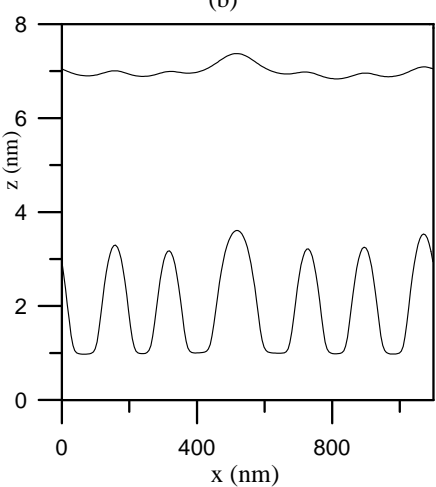

(d)

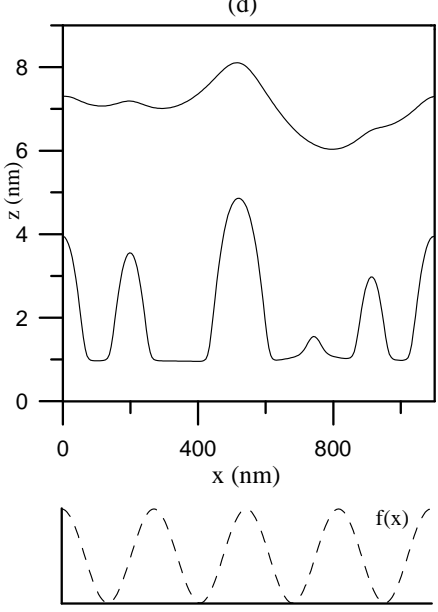

FIGURE 12. Time frames of bilayer evolution with active laser beam interference towards embedded nanoparticles. $h_{10}=2 \mathrm{~nm}, h_{20}=7 \mathrm{~nm}$. (a) $t=0.44 \times 10^{-4} \mathrm{~s}$, (b) $t=$ $0.66 \times 10^{-4} \mathrm{~s}$, (c) $t=0.57 \times 10^{-3} \mathrm{~s}$, (d) $t=0.76 \times 10^{-3} \mathrm{~s}$.

\section{References}

[1] A. Vrij, J. Th. G. Overbeek. Rupture of Thin Liquid Films Due to Spontaneous Fluctuations in Thickness. J. Am. Chem. Soc., 90 (1968), 3074-3078.

[2] G. Reiter. Dewetting of thin polymer films. Phys. Rev. Lett., 68 (1992), 75-78.

[3] A. Sharma, R. Khanna. Pattern Formation in Unstable Thin Liquid Films. Phys. Rev. Lett., 81 (1998), 3463-3466.

[4] R.M. Bradley, J.M.E. Harper. Theory of ripple topography induced by ion bombardment. J. Vac. Sci. Tech. A, 6 (1988), 2390-2395.

[5] E. Chason, T.M. Mayer, B.K. Kellerman, D.T. Mcllroy, A.J. Howard. Roughening instability and evolution of the Ge(001) surface during ion sputtering. Phys. Rev. Lett., 72 (1994), 3040-3043.

[6] J. Bischof, D. Scherer, S. Herminghaus, P. Leiderer. Dewetting Modes of Thin Metallic Films: Nucleation of Holes and Spinodal Dewetting. Phys. Rev. Lett., 77 (1996), 1536-1539.

[7] S.J. Henley, J.D. Carey, S.R.P. Silva. Pulsed-laser-induced nanoscale island formation in thin metal-on-oxide films. Phys. Rev. B, 72 (2005), 195408-18.

[8] J. Trice, D. Thomas, C. Favazza, R. R. Sureshkumar, R. Kalyanaraman. Investigation of pulsed laser induced dewetting in nanoscopic metal films. Phys. Rev. B, 75 (2007), 235439-54.

[9] C. Zhang, R. Kalyanaraman. In-situ nanostructured film formation during physical vapor deposition. Appl. Phys. Lett., 83 (2003), 4827-4829.

[10] C. Favazza, J. Trice, A.K. Gangopadhyay, H. Garcia, R. Sureshkumar, R. Kalyanaraman. Nanoparticle ordering by dewetting of Co on SiO2. J. Electron. Mater., 35 (2006), 1618-1620. 
[11] C. Favazza, R. Kalyanaraman, R. Sureshkumar. Robust nanopatterning by laser-induced dewetting of metal nanofilms. Nanotechnology, 17 (2006), 4229-4234.

[12] J. Trice, C. Favazza, D. Thomas, H. Garcia, R. Kalyanaraman, R. R. Sureshkumar. Novel self-organization mechanism in ultrathin liquid films: theory and experiment. Phys. Rev. Lett., 101 (2008), 017802-6.

[13] H. Krishna, R. Sachan, J. Strader, C. Favazza, M. Khenner, R. Kalyanaraman. Thickness-dependent spontaneous dewetting morphology of ultrathin Ag films. Nanotechnology, 21 (2010), 155601-8.

[14] L. Longstreth-Spoor, J. Trice, H. Garcia, C. Zhang, R. Kalyanaraman. Nanostructure and microstructure of laserinterference-induced dynamic patterning of Co on Si. J. Phys. D: Appl. Phys., 39 (2006), 5149-5159.

[15] C. Favazza, J. Trice, R. Kalyanaraman, R. Sureshkumar. Self-organized metal nanostructures through laserinterference driven thermocapillary convection. Appl. Phys. Lett., 91 (2007), 043105-7.

[16] H. Krishna, N. Shirato, S. Yadavali, R. Sachan, J. Strader, R. Kalyanaraman. Self-organization of nanoscale multilayer liquid metal films: Experiment and theory. ACS Nano, 5 (2011), 470-476.

[17] F. Brochard-Wyart, P. Martin, C. Redon. Liquid/liquid dewetting. Langmuir, 9 (1993), 3682-3690.

[18] P. Lambooy, K.C. Phelan, O. Haugg, G. Krausch. Dewetting at the Liquid-Liquid Interface. Phys. Rev. Lett., 76 (1996), 1110-1113.

[19] M. Sferrazza, M. Heppenstall-Butler, R. Cubitt, D. Bucknall, J. Webster, R. A. L. Jones. Interfacial Instability Driven by Dispersive Forces: The Early Stages of Spinodal Dewetting of a Thin Polymer Film on a Polymer Substrate. Phys. Rev. Lett., 81 (1998), 5173-5176.

[20] M.O. David, G. Reiter, T. Sitthai, J. Schultz. Deformation of a Glassy Polymer Film by Long-Range Intermolecular Forces. Langmuir, 14 (1998), 5667-5672.

[21] R.A. Segalman, P.F. Green. Dynamics of Rims and the Onset of Spinodal Dewetting at Liquid/Liquid Interfaces. Macromolecules, 32 (1999), 801-807.

[22] C. Wang, G. Krausch, M. Geoghegan. Dewetting at a Polymer-Polymer Interface: Film Thickness Dependence. Langmuir, 17 (2001), 6269-6274.

[23] J.P. de Silva, M. Geoghegan, A.M. Higgins, G. Krausch, M.O. David, G. Reiter. Switching Layer Stability in a Polymer Bilayer by Thickness Variation. Phys. Rev. Lett., 98 (2007), 267802-5.

[24] L. Xu, T. Shi, L. An. The competition between the liquid-liquid dewetting and the liquid-solid dewetting. J. Chem. Phys., 130 (2009), 184903-10.

[25] A. Pototsky, M. Bestehorn, D. Merkt, U. Thiele. Alternative pathways of dewetting for a thin liquid two-layer film. Phys. Rev. E, 70 (2004), 025201-4.

[26] A. Pototsky, M. Bestehorn, D. Merkt. Morphology changes in the evolution of liquid two-layer films. J. Chem. Phys., 122 (2005), 224711-23.

[27] D. Bandyopadhyay, R. Gulabani, A. Sharma. Instability and dynamics of thin liquid bilayers. Ind. Eng. Chem. Res., 44 (2005), 1259-1272.

[28] L.S. Fisher, A.A. Golovin. Nonlinear stability analysis of a two-layer thin liquid film: Dewetting and authophobic behavior. J. Colloid Interface Science, 291 (2005), 515-528.

[29] D. Merkt, A. Pototsky, M. Bestehorn, U. Thiele. Long-wave theory of bounded two-layer films with a free liquid-liquid interface: Short- and long-time evolution. Phys. Fluids, 17 (2005), 064104-23.

[30] A. Pototsky, M. Bestehorn, D. Merkt, U. Thiele. Evolution of interface patterns of three-dimensional two-layer liquid films. Europhys. Lett., 74 (2006), 665-671.

[31] D. Bandyopadhyay, A. Sharma. Nonlinear instabilities and pathways of rupture in thin liquid bilayers. J. Chem. Phys., 125 (2006), 054711-13.

[32] A.A. Nepomnyashchy, I. B. Simanovskii. Decomposition of a two-layer thin liquid film flowing under the action of Marangoni stresses. Phys. Fluids, 18 (2006), 112101-11.

[33] A.A. Nepomnyashchy, I.B. Simanovskii. Marangoni instability in ultrathin two-layer films. Phys. Fluids, 19 (2007), 122103-14

[34] A.A. Nepomnyashchy, I.B. Simanovskii. The Influence of Gravity on the Dynamics of Non-Isothermic Ultra-Thin Two-Layer Films. Microgravity Sci. Technol., 21 (2009), S261-S269.

[35] B.B. Yellen, O. Hovorka, G. Friedman. Arranging matter by magnetic nanoparticle assemblers. Proc. Nat. Acad. Sci., $102(2005), 8860-8864$.

[36] M.A.M. Gijs. Magnetic bead handling on-chip: new opportunities for analytical applications. Microfluidics and Nanofluidics, 1 (2004), 22-40.

[37] Y.M. Hao, M. Chen, Z.B. Hu. Effective removal of Cu(II) ions from aqueous solution by amino-functionalized magnetic nanoparticles. J. Hazard. Mat., 184 (2010), 392-399.

[38] J. Wang, L.Y. Wang, Y. Sun, X.N. Zhu, H.Y. Xu, N. Bi, H.Q. Zhang, Y.B. Cao, X.H. Wang, D.Q. Song. Preparation of core/shell Fe3O4/Au nanocomposite and its application to surface plasmon resonance biosensor. Acta Chimica Sinica, 68 (2010), 263-268.

[39] B. Sepúlveda, A. Calle, L.M. Lechuga, G. Armelles. Highly sensitive detection of biomolecules with the magneto-optic surface-plasmon-resonance sensor. Opt. Lett., 31 (2006), 1085-1087.

[40] D.M. Newman, R.J. Matelon, M.L. Wears, L.B. Savage. The In Vivo Diagnosis of Malaria: Feasibility Study Into a Magneto-Optic Fingertip Probe. IEEE J. Sel Top. Quant. Elec., 16 (2010), 573-580.

[41] R. Bahuguna, M. Mina, R.J. Weber. Mach-Zehnder interferometric switch utilizing Faraday rotation. IEEE Trans. Mag., 43 (2007), 2680-2682.

[42] L. Eldada. Optical communication components. Rev. Sci. Instrum., 75 (2004), 575-593. 
[43] K. Yang, C. Clavero, J. R. Skuza, M. Varela, R. A. Lukaszew. Surface plasmon resonance and magneto-optical enhancement on Au-Co nanocomposite thin films. J. Appl. Phys., 107 (2010), 103924-5.

[44] P.K. Jain, Y. Xiao, R. Walsworth, A.E. Cohen. Surface Plasmon Resonance Enhanced Magneto-Optics (SuPREMO): Faraday Rotation Enhancement in Gold-Coated Iron Oxide Nanocrystals. Nano Lett., 9 (2009), 1644-1650.

[45] N. Pazos-Perez, Y. Gao, M. Hilgendorff, S. Irsen, J. Pereez-Juste, M. Spasova, M. Farle, L.M. Liz-Marzan, M. Giersig Magnetic-noble metal nanocomposites with morphology-dependent optical response. Chem. Mat., 19 (2007), 4415-4422.

[46] V.S. Ajaev, D.A. Willis. Thermocapillary flow and rupture in films of molten metal on a substrate. Phys. Fluids, 15 (2003), 3144-7; Heat transfer, phase change, and thermocapillary flow in films of molten metal on a substrate. Numer. Heat Transfer, Part A, 50 (2006), 301-313.

[47] A.S. Basu, Y.B. Gianchandani. Shaping high-speed Marangoni flow in liquid films by microscale perturbations in surface temperature. Appl. Phys. Lett., 90 (2007), 034102-3.

[48] F.J. Higuera. Steady thermocapillary-buoyant flow in an unbounded liquid layer heated nonuniformly from above. Phys. Fluids, 12 (2000), 2186-12.

[49] A. Oron, Y. Peles. Stabilization of thin liquid films by internal heat generation. Phys. Fluids, 10 (1998), 537-3.

[50] A. Oron. Nonlinear dynamics of irradiated thin volatile liquid films. Phys. Fluids, 12 (2000), 29-13.

[51] R.O. Grigoriev. Control of evaporatively driven instabilities of thin liquid films. Phys. Fluids, 14 (2002), $1895-15$.

[52] L. Kondic, J.A. Diez, Philip D. Rack, Yingfeng Guan, Jason D. Fowlkes. Nanoparticle assembly via the dewetting of patterned thin metal lines: Understanding the instability mechanisms. Phys. Rev. E, 79 (2009), 026302-7.

[53] Y. Wu, J. D. Fowlkes, P. D. Rack, J. A. Diez, L. Kondic. On the Breakup of Patterned Nanoscale Copper Rings into Droplets via Pulsed-Laser-Induced Dewetting: Competing Liquid-Phase Instability and Transport Mechanisms. Langmuir, 26 (2010), 11972-11979.

[54] Y. Wu, J. D. Fowlkes, N. A. Roberts, J. A. Diez, L. Kondic, A. G. Gonzalez, P. D. Rack. Competing liquid phase instabilities during pulsed laser induced self-assembly of copper rings into ordered nanoparticle arrays on SiO2. Langmuir, 27 (2011), 13314-13323.

[55] H. Krishna, N. Shirato, C. Favazza, R. Kalyanaraman. Energy driven self-organization in nanoscale metallic liquid films. Phys. Chem. Chem. Phys., 11 (2009), 8136-8143.

[56] A. Atena, M. Khenner. Thermocapillary effects in driven dewetting and self-assembly of pulsed-laser-irradiated metallic films. Phys. Rev. B, 80 (2009), 075402-11.

[57] A. Oron, S.H. Davis, S.G. Bankoff. Long scale evolution of thin liquid films. Rev. Mod. Phys., 69 (1997), 931-980.

[58] M. Khenner, S. Yadavali, R. Kalyanaraman. Formation of organized nanostructures from unstable bilayers of thin metallic liquids, Phys. Fluids, 23 (2011), 122105-14.

[59] C. Favazza, R. Kalyanaraman, R. Sureshkumar. Dynamics of ultrathin metal films on amorphous substrates under fast thermal processing. J. Appl. Phys., 102 (2007), 104308-6.

[60] B.V. Derjaguin, L.F. Leonov, V.I. Roldughin. Disjoining pressure in liquid metallic films. J. Colloid Interface Sci., 108 (1985), 207-214; also in: Prog. Surf. Sci. 40 (1992), 232-239.

[61] S. Yadavali, R. Kalyanaraman. Morphology transitions in ternary dewetting systems. Submitted.

[62] S. Yadavali, R. Kalyanaraman. Thermal modeling for multilayer thin films using pulsed laser induced dewetting. In preparation.

[63] J.S.C. Prentice. Coherent, partially coherent and incoherent light absorption in thin-film multilayer structures. J. Phys. D: Appl. Phys., 33 (2000), 3139-3145.

[64] S.H. Davis. On the principle of exchange of stabilities. Proc. Roy. Soc. Ser. A, 310 (1969), 341-358.

[65] V.M. Starov, M.G. Velarde, C.J. Radke. Wetting and Spreading Dynamics. CRC, Boca Raton, 2007.

[66] J. Israelachvili. Intermolecular and Surface Forces. Academic, London, 1991.

[67] E. Hairer, G. Wanner. Stiff differential equations solved by Radau method. J. Comput. Appl. Math., 111 (1999), 93-111.

[68] P. N. Brown, G. D. Byrne, A. C. Hindmarsh. VODE: A variable coefficient ODE solver. SIAM J. Sci. Stat. Comput., 10 (1989), 1038-1051.

[69] M.H. Ward. Interfacial thin films rupture and self-similarity. Phys. Fluids, 23 (2011), 062105-14.

[70] K. Glasner, T. Witelski. Coarsening dynamics of dewetting films. Phys. Rev. E, 67 (2003), 016302-12. 